\title{
Una revisión de la asimetría bilateral en bivalvos
}

\author{
A review of bilateral asymmetry in bivalves
}

\section{Carolina Briones $^{1}$ y Ricardo Guiñez ${ }^{2}$}

\author{
${ }^{1}$ Programa Doctorado en Ciencias Aplicadas, mención Sistemas Marinos Costeros, Facultad de Recursos del Mar, \\ Universidad de Antofagasta, Casilla 170, Antofagasta, Chile \\ ${ }^{2}$ Laboratorio de Evolución Experimental, Instituto de Investigaciones Oceanológicas, Facultad de Recursos del Mar, \\ Universidad de Antofagasta, Casilla 170, Antofagasta, Chile \\ rguinez@uantof.cl
}

\begin{abstract}
During the last years, studies on bilateral asymmetry have substantially increased. However, in the literature exists a clear trend toward the study of the fluctuating asymmetry over directional asymmetry and anti symmetry, because it has been assumed that the first one reflects the developmental instability facing environmental or genetic disruptions. Anyway, it is important to study both directional asymmetry and anti symmetry, because they would provide information about ontogenetic and evolutionary processes of fluctuating asymmetry. Only exploratory studies have been made in bivalves in spite of their potential use as a tool in monitoring the stress levels in natural populations and as an indicator of the Darwinian fitness. In this review the significance of the bivalve bilateral asymmetry and factors that regulate the asymmetric components in an evolutionary context are enhanced. We emphasize the necessity of simultaneous studies of the three types of bilateral asymmetry at different geographical scales in the range of the distribution of the species, to determine the ecological and evolutionary patterns and the main factors that cause it.
\end{abstract}

Key words: Mollusks, bilateral symmetry, fluctuating asymmetry, directional asymmetry

\section{Introducción}

Uno de los problemas centrales en estudios de biología comparada es el cambio evolutivo de los mecanismos del desarrollo ontogénico (Klingenberg et al. 2001). Este cambio se considera adaptativo y, por lo tanto, sensible a procesos de selección natural. Es esperable entonces que algunas variantes de los procesos del desarrollo ontogénico puedan producir cambios en las estructuras morfológicas y en la función del organismo para que sean favorecidas por la selección natural. Para una mejor comprensión del aspecto adaptativo de la evolución del desarrollo ontogénico, es de crucial importancia reconsiderar la relación entre los procesos del desarrollo ontogénico, las características morfológicas y la función resultante en los individuos y en su eficacia biológica.
Resumen.- En los últimos años se ha observado un incremento en las investigaciones de la asimetría bilateral. Sin embargo, de los tipos de asimetría bilateral descritos (i.e. asimetría fluctuante, direccional y antisimetría) existe una notable tendencia en la literatura hacia el estudio de la asimetría fluctuante, puesto que se presume que ésta reflejaría la inestabilidad del desarrollo frente a perturbaciones de origen ambiental o genético. No obstante, también es importante estudiar la asimetría direccional y la antisimetría ya que ellas indicarían procesos de cambio ontogénico evolutivo de la asimetría fluctuante. En bivalvos, estudios de esta índole han sido poco explorados, pese a su uso potencial como una herramienta para el seguimiento de los niveles de estrés en las poblaciones naturales y como un indicador de la calidad individual o de la adecuación darwiniana. En esta revisión se resalta la importancia del estudio de la asimetría bilateral en bivalvos, y los factores que regularían los diferentes componentes asimétricos en un contexto evolutivo. Se destaca la necesidad de estudiar simultáneamente los tres tipos de asimetría bilateral a diferentes escalas geográficas dentro del rango de distribución de las especies, de tal modo de poder determinar sus patrones ecológicos y evolutivos y sus factores causales.

Palabras clave: Moluscos, simetría bilateral, asimetría fluctuante, asimetría direccional
Es al nivel de la expresión y la función morfológica, que los cambios del desarrollo ontogénico tienen consecuencias para el ajuste del organismo, siendo crítico este nivel para establecer la unión entre los procesos del desarrollo ontogénico y el de la evolución (Palmer 1994). Un ámbito en que esto último puede ser abordado, tiene relación con la expresión de la simetría bilateral (SB). La SB ocurre en rasgos del cuerpo bilateralmente simétricos en que la expresión de su lado izquierdo debiera ser una imagen especular de su lado derecho. Esta simetría puede presentar variaciones menores o mayores durante el desarrollo ontogénico que resultan en asimetrías entre los lados izquierdo y derecho (Fig. 1) (Palmer \& Strobeck 1986). La asimetría entre los lados del cuerpo se encuentra a su vez íntimamente relacionada 

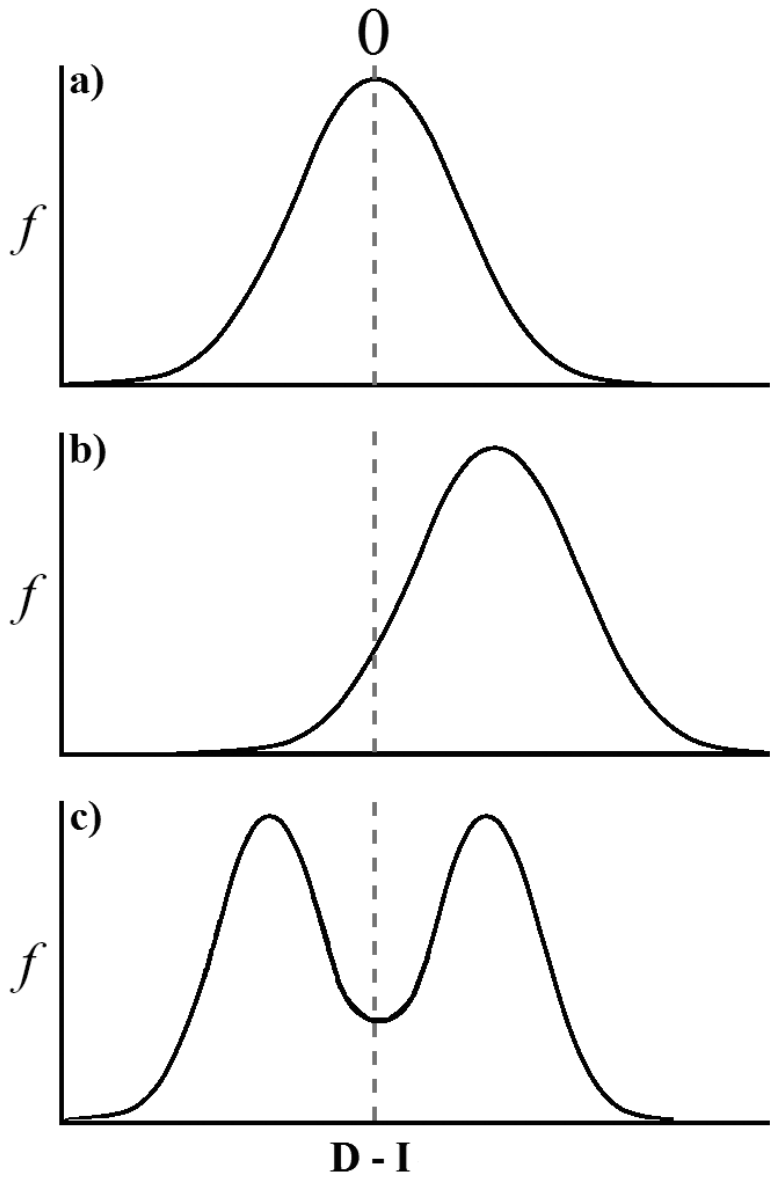

Figura 1

Tres distribuciones de frecuencias comunes para las diferencias entre los valores del lado derecho (D) y el izquierdo (I) de un rasgo bilateral, expresadas como la diferencia D - I: a) asimetría fluctuante, b) asimetría direccional y c) antisimetría. Después de Palmer (1994)

Three common frequency distributions of the differences between the measured values of the right (D) and the left (I) side from a bilateral trait, expressed as the difference between $\mathrm{D}$ - I: a) fluctuating asymmetry, b) directional asymmetry, and c) antisymmetry. After Palmer (1994)

con la estabilidad del desarrollo, que es la habilidad de un organismo para desarrollar un fenotipo consistente en un ambiente determinado y que refleja tanto la adaptación al ambiente experimentada durante el desarrollo, como la coadaptación entre los genes (Graham et al. 2003).

\section{Asimetría bilateral}

Se han descrito diferentes tipos de asimetría bilateral (AB). Entre ellas, la asimetría fluctuante (AF), que corresponde a desviaciones sutiles y aleatorias de la simetría en rasgos bilateralmente simétricos (Fig. 1a) (Palmer \& Strobeck 2003), la asimetría direccional (AD), que ocurre cuando el valor de un lado del rasgo es más grande que el otro (distribución normal con promedio distinto de cero), incluyendo situaciones en que estas diferencias son sutiles (Fig. 1b); por último, la antisimetría (AS), que corresponde al caso en que los rasgos presentan una distribución bimodal con promedio cero (Fig.1c) (Palmer \& Strobeck 1986).

Un número notable de diferentes índices de asimetría ha sido utilizado en estudios previos de AB (Palmer \& Strobeck 1986). Sin embargo, la AB puede estimarse como el valor absoluto de la diferencia $D_{i}-I_{i}$, donde $D_{i}$ representa el valor del rasgo del lado o valva derecha, $I_{i}$ es el respectivo valor del lado izquierdo, para el 'i' individuo (Palmer \& Strobeck 2003).

\section{Importancia del estudio de la asimetría}

La AF es la medida de la inestabilidad del desarrollo más ampliamente usada (Graham et al. 2003) y ha sido definida como una medida de la desviación de la simetría bilateral perfecta, en relación con el desarrollo de características morfológicas que normalmente son simétricas (van Valen 1962, Ditchkoff et al. 2001), puesto que estimaría la incapacidad de los organismos para mantener su desarrollo normal, contra ruidos o perturbaciones de origen ambiental (e.g. temperaturas extremas, contaminantes, malnutrición, parásitos) o genético (e.g. estrés asociado a procesos de selección intensa, endogamia/exogamia, mutaciones) (Palmer \& Strobeck 1986, Palmer \& Strobeck 1992, Lens \& van Dongen 2000, Rasmuson 2002). Sin embargo, las causas ontogénicas de las diferencias bilaterales son desconocidas en muchos grupos, por lo que es necesario evaluar el impacto relativo de los factores genéticos y los ambientales, sobre la evolución de la asimetría (Palmer 1996a). La asimetría direccional, por otra parte, se considera con una base genética significativa (Palmer \& Strobeck 1986, Palmer 1994) y, por lo tanto, no sería apropiada para medir la inestabilidad del desarrollo, pero sí para la estimación de procesos adaptativos. Lo anterior puede explicar una tendencia en la literatura de la inestabilidad del desarrollo hacia el estudio de la asimetría fluctuante. Sin embargo, existe evidencia creciente de que los tres tipos de asimetría debieran ser considerados como procesos dinámicamente interrelacionados (Lens \& van Dongen 2000). 


\section{Algunas ventajas del estudio de asimetrías}

Los rasgos bilateralmente simétricos son muy comunes en la naturaleza y la medición de la AF ha surgido como un método relativamente simple para la evaluación del estrés biológico de los individuos. Debido a lo anterior es que la AF se ha propuesto como una herramienta de utilidad en programas de monitoreo, de conservación biológica y de estudios de la estabilidad del desarrollo (Tomkins \& Kotiaho 2001, Fréchette \& Daigle 2002). Por otra parte, el hecho de que la AF aparezca negativamente correlacionada con la aptitud biológica, abre la posibilidad de estudiar experimentalmente el modo en que la selección natural estaría actuando sobre el rasgo y al mismo tiempo, evaluar si el rasgo tiene heredabilidad significativa como para responder a las presiones selectivas y ser incluido en programas de mejora genética (Manning \& Ockenden 1994).

La AS y la AD pueden ser indicadoras de procesos de cambio ontogénico evolutivo de la AF, pero de cuyas causas se conoce muy poco (Palmer 1994). Entre las pocas causas reconocidas se han sugerido mecanismos genéticos, que ocurren cuando se rompen o desacoplan los sistemas coadaptados de genes que regulan la canalización de los rasgos bilaterales (Palmer \& Strobeck 1986). Llama la atención que a la fecha la implicancia evolutiva de la antisimetría, siendo un tipo tan peculiar de variación, haya sido tan poco considerada y valorada (Palmer 2005). Cabe destacar que una presunción común en la literatura de la estabilidad del desarrollo es que el desarrollo de los lados izquierdo y derecho procede independientemente. Sin embargo, durante la ontogenia, la estabilidad del desarrollo puede implicar un control de retroalimentación entre los dos lados de un rasgo, lo cual lleva a mecanismos de crecimiento compensatorio diferencial entre ambos lados, que reduciría la asimetría inicial durante el desarrollo (Hoffmann et al. 1999).

\section{Evolución, asimilación genética y asimetría}

La virtual ausencia de variación heredable para la dirección de la asimetría en especies antisimétricas y, por otra parte, la casi universal herencia de la dirección en especies direccionalmente asimétricas, provee una oportunidad única para examinar la frecuencia de dos modos de evolución fundamentalmente diferentes: la evolución convencional, en donde la variación fenotípica original surge inicialmente de mutaciones azarosas de pequeño efecto (i.e. la variación genética precede a la variación fenotípica observable), y la asimilación genética, donde la variación fenotípica original surge inicialmente desde el ambiente durante el desarrollo y donde las mutaciones que canalizan estos fenotipos novedosos surgen más tarde evolutivamente (i.e. variación genética posterior a la variación fenotípica observable) (Palmer 2005). Por este motivo, los cambios filogenéticos entre los estados de asimetría permiten una poderosa prueba de la frecuencia de estos dos modos de evolución, puesto que las dos alternativas evolutivas para la AD son posible (Palmer 1994). Por la primera vía, taxa direccionalmente asimétricos deberían surgir directamente de ancestros simétricos. Sin embargo, la segunda vía parece ser más interesante, puesto que si los primeros linajes derivados de un ancestro simétrico fueron antisimétricos, y los taxa direccionalmente asimétricos derivaron de estos, entonces la AS (dirección no heredable) precedió a la AD (dirección heredable) evolutivamente (Palmer 1994, 2005).

\section{Patrones geográficos}

En otro contexto, la caracterización de procesos que operan en diferentes escalas espaciales (e.g. micro, meso y macroescala) y temporales, y cómo éstos interactúan para formar patrones ecológicos, ha sido el foco central en la investigación ecológica contemporánea (Levin 1992, Broitman et al. 2001). Asimismo, en especies distribuidas sobre un amplio rango latitudinal, la variación geográfica en rasgos morfológicos y de historia de vida, puede reflejar relaciones muy diferentes de los efectos genotípicos y ambientales (Via et al. 1995). Debido a lo anterior, la posibilidad de analizar la AB y sus componentes (i.e. AF y AD) en la macroescala geográfica, puede permitir avances significativos en torno a esta problemática. Al respecto, solo un estudio realizado en poblaciones naturales de peces de la especie Gasterosteus aculeatus (Bergstrom \& Reimchen 2002, 2003), aporta evidencias de variación en la distribución geográfica de la asimetría que refleja la importancia relativa de rasgos bilaterales, que conforman el aparato de defensas contra la depredación, para sobrevivir en diferentes hábitat. Este estudio permite destacar la utilidad de la $\mathrm{AB}$ para inferir el impacto que los rasgos bilaterales pueden tener sobre la eficacia biológica. La reducción de la eficacia biológica en individuos asimétricos podría ser una consecuencia funcional directa de la asimetría y reflejaría la inestabilidad del desarrollo (Bergstrom \& Reimchen 2003).

\section{Asimetría bilateral en bivalvos}

Entre los moluscos, la antisimetría pura es la más común dentro de los bivalvos. Las conchas desiguales son más bien desarrolladas en taxa bivalvas en las cuales una valva está fijada al sustrato y la otra permanece libre para moverse (Palmer 2005). En un contexto evolutivo, los registros fósiles de bivalvos adheridos al sustrato, indican 
que la transición [simetría $\rightarrow$ asimetría direccional] ha ocurrido dos veces y la transición [simetría $\rightarrow$ antisimetría $\rightarrow$ asimetría direccional] al menos tres veces (Palmer 1996a).

Asimismo, se ha documentado que en moluscos bivalvos existen grupos con AD conspicua; por ejemplo, los individuos pueden presentar la valva con la que se adhieren al sustrato más pequeña (e.g. Anomiacea y Pectinacea) o de mayor tamaño (e.g. Ostreacea) (Palmer 1996b). Sin embargo, en el chorito maico Perumytilus purpuratus se ha encontrado evidencia de AD más sutil, con la valva derecha consistentemente más alongada que la izquierda (Briones \& Guiñez 2005). En este último caso, la evidencia experimental sugiere que la asimetría podría estar siendo modulada por la densidad o la estratificación de los choritos, los individuos localizados en el estrato superior se ven menos afectados por la competencia intraespecífica que aquellos ubicados en estratos inferiores, presentando además diferencias en la distribución de tamaños y en la cantidad de reclutas (Alvarado \& Castilla 1996, Guiñez \& Castilla 1999). En otros casos como en el pectínido Chlamys islandica y en la ostra japonesa Crassostrea gigas, se ha documentado casos de AF asociados a diferentes tasas de mortalidad (Fréchette \& Daigle 2002, Fréchette et al. 2003). Sin embargo, en Dreissena polymorpha, especie utilizada extensamente en estudios de bioacumulación como monitor de contaminación, Voets et al. (2006) no encontraron evidencia significativa de $\mathrm{AB}$ en las valvas, como consecuencia del impacto de contaminantes, concluyéndose que la contaminación no representaría un agente estresante en estos organismos.

No obstante, cómo la AF o eventualmente la AD puede cambiar en bivalvos en el ámbito geográfico (e.g. latitudinal) sólo ha sido explorada recientemente en mitílidos ${ }^{1}$, siendo en general, este tipo de problemática de muy reciente interés.

\section{Perspectivas}

Existe poco conocimiento acerca de las transiciones entre los tipos de asimetrías descritos, especialmente en sistemas marinos costeros y en poblaciones naturales. A menudo se asume que la AD y la AS tienen una mayor base genética que la $\mathrm{AF}$, lo que ha llevado a muchos científicos a excluir, en estudios de la inestabilidad del desarrollo, a rasgos o poblaciones que muestran cualquiera de estos dos tipos de asimetrías, focalizando

${ }^{1}$ Briones C, R Guiñez, JE Toro, M Astorga, P Presa \& M Pérez. 2007. Asimetría bilateral de la forma y el grosor de las valvas en el chorito maico Perumytilus purpuratus. XII Congreso Latinoamericano de Ciencias del Mar, Florianópolis, Brasil. toda la atención sólo en la AF. Estos procedimientos pueden desviar nuestra búsqueda y, de esta manera, nuestro entendimiento acerca de los patrones de la asimetría bilateral y de los factores que la afectan (Kark 2001).

El desarrollo de un rasgo bilateral de un individuo se encuentra presumiblemente bajo la influencia de los mismos genes o de un complejo de genes (Clarke 1993). Sin embargo, se ha sugerido que la desviación desde la simetría hacia los lados derecho o izquierdo no tiene una base genética significante y representa una variación de origen ambiental (Palmer \& Strobeck 1992). En la literatura se ha propuesto la hipótesis de que la asimetría de rasgos bilaterales sería un reflejo de la inestabilidad del desarrollo, por lo que la asimetría debiera incrementar en la medida que incrementa el estrés o perturbaciones de origen ambiental o genético (Palmer \& Strobeck 1992, Lens \& van Dongen 2000).

De la extensa literatura existente en AB (Bergstrom \& Reimchen 2002), los estudios en moluscos bivalvos descritos arriba han sido realizados en condiciones de escala local, lo que a la fecha no permite aún tener una visión de la variación en la meso y la macroescala geográfica. Al respecto, sólo en el estudio de Briones ${ }^{1} \mathrm{y}$ en el contexto de macroescala a nivel latitudinal, se muestran evidencias en un molusco, que la variación de la AF y la AD estaría en parte modulada por la estratificación y la densidad para los rasgos de grosor y forma de las valvas. Los resultados muestran que la asimetría de ambos rasgos responde de modo diferencial a la densidad, pero del mismo modo a la estratificación de los individuos dentro de las matrices.

A la luz de esta revisión recomendamos que en los futuros estudios de rasgos de $\mathrm{AB}$ y de la inestabilidad del desarrollo, debiera trabajarse simultáneamente cuando sea posible- con las tres formas de asimetría, o al menos con las dos más comunes (AF y AD), de tal modo de poder generar un mayor conocimiento sobre las transiciones e interconexiones entre las diferentes formas de asimetría. Lo que puede permitir poner a prueba la relación y dinámica de la interfase entre los distintos tipos de asimetrías en las distintas escalas geográficas (Kark 2001). Esto, junto a la necesidad de implementar experimentos manipulativos, puede permitir avanzar en la comprensión de los mecanismos subyacentes de la asimetría direccional y fluctuante en bivalvos, en particular, de aquellas especies que, como los mitílidos tienden a ser dominantes y gregarios (Guiñez \& Castilla 1999, Briones \& Guiñez 2005). Estudios experimentales en distintas escalas geográficas permitirán avanzar en la dilucidación de los mecanismos selectivos, ecológicos y fisiológicos que determinan y 
modulan a los rasgos con asimetría bilateral, en particular, los relacionados con la vida gregaria, la adecuación darwiniana y su expresión en el ámbito geográfico de distribución de la especie y sus posibles implicancias evolutivas.

\section{Agradecimientos}

Agradecemos a F. Concha, A. Pacheco y dos revisores anónimos por la lectura crítica y sugerencias realizadas sobre una versión previa del manuscrito, y a N. Fuentes por traducir el resumen. Finalmente, agradecemos el financiamiento del proyecto FONDECYT 1050848 a RG.

\section{Literatura citada}

Alvarado JL \& JC Castilla. 1996. Tridimensional matrices of Perumytilus purpuratus on intertidal platforms with varying wave forces in central Chile. Marine Ecology Progress Series 133: 135-141.

Bergstrom CA \& TE Reimchen. 2002. Geographical variation in asymmetry in Gasterosteus aculeatus. Biological Journal of the Linnean Society 77(1): 9-22.

Bergstrom CA \& TE Reimchen. 2003. Asymmetry in structural defenses: Insights into selective predation in the wild. Evolution 57(9): 2128-2138.

Briones C \& R Guiñez. 2005. Asimetría bilateral de la forma de las valvas y posición espacial en matrices del chorito Perumytilus purpuratus (Lamarck, 1819) (Bivalvia: Mytilidae). Revista Chilena de Historia Natural 78(1): 314.

Broitman BR, SA Navarrete, F Smith \& SD Gaines. 2001. Geographic variation of southeastern Pacific intertidal communities. Marine Ecology Progress Series 224: 2134.

Clarke GM. 1993. The genetic basis of developmental stability. I. Relationships between stability, heterozygosity and genomic coadaptation. Genetica 89(1-3): 15-23.

Ditchkoff SS, RL Lochmiller, RE Masters, SR Hoofer \& RA van Den Bussche. 2001. Major-histocompatibilitycomplex-associated variation in secondary sexual traits of white-tailed deer (Odocoileus virginianus): Evidence for good-genes advertisement. Evolution 55(3): 616-625.

Fréchette M \& G Daigle. 2002. Growth, survival and fluctuating asymmetry of Iceland scallops in a test of density-dependent growth in a natural bed. Journal of Experimental Marine Biology and Ecology 270(1): 73-91.

Fréchette M, P Goulletquer \& G Daigle. 2003. Fluctuating asymmetry and mortality in cultured oysters (Crassostrea gigas) in Marennes-Oléron basin. Aquatic Living Resources 16(4): 339-346.

Graham JH, K Shimizu, JM Emlen, DC Freeman \& J Merkel. 2003. Growth models and the expected distribution of fluctuating asymmetry. Biological Journal of the Linnean Society 80(1): 57-65.

Guiñez R \& JC Castilla. 1999. A tridimensional self-thinning model for multilayered intertidal mussels. American Naturalist 154(3): 341-357.

Hoffmann AA, RE Woods \& GM Clarke. 1999. A comment on in-group/out-group comparisons for fluctuating asymmetry based on trait values from the left or right sides of an individual. American Naturalist 153(1): 140-142.

Kark S. 2001. Shifts in bilateral asymmetry within a distribution range: The case of the chukar partridge. Evolution 55(10): 2088-2096.

Klingenberg CP, AV Badyaev, SM Sowry \& NJ Beckwith. 2001. Inferring developmental modularity from morphological integration: Analysis of individual variation and asymmetry in bumblebee wings. American Naturalist 157(1): 11-23.

Lens L \& S van Dongen. 2000. Fluctuating and directional asymmetry in natural bird populations exposed to different levels of habitat disturbance, as revealed by mixture analysis. Ecology Letters 3(6): 516-522.

Levin SA. 1992. The problem of pattern and scale in ecology. The Robert H. MacArthur Award Lecture. Ecology 73(6): 1943-1967.

Manning JT \& L Ockenden. 1994. Fluctuating asymmetry in racehorses. Nature 370: 185-186.

Palmer AR. 1994. Fluctuating asymmetry analyses: A primer. En: Markow TA(ed). Developmental Instability: Its origins and evolutionary implications, pp. 335-364. Kluwer, Dordrecht.

Palmer AR. 1996a. From symmetry to asymmetry: Phylogenetic patterns of asymmetry variation in animals and their evolutionary significance. Proceedings of the National Academy of Sciences of the United States of America 93(25): 14279-14286.

Palmer AR. 1996b. Waltzing with asymmetry. Is fluctuating asymmetry a powerful new tool for biologists or just an alluring new dance step? BioScience 46(7): 518-532.

Palmer AR. 2005. Antisymmetry. En: Hallgrimmson B \& BK Hall (ed). Variation, pp. 359-397. Elsevier, Amsterdam.

Palmer AR \& C Strobeck. 1986. Fluctuating asymmetry: measurement, analysis, patterns. Annual Review of Ecology and Systematics 17: 391-421.

Palmer AR \& C Strobeck. 1992. Fluctuating asymmetry as a measure of developmental stability: Implications of nonnormal distributions and power of statistical tests. Acta Zoologica Fennica 191: 57-72.

Palmer AR \& C Strobeck. 2003. Fluctuating asymmetry analyses revisited. En: Polak M (ed). Developmental Instability. Causes and consequences, pp. 279-319. Oxford University Press, Oxford. 
Rasmuson M. 2002. Fluctuating asymmetry - indicator of what? Hereditas 136(3): 177-183.

Tomkins JL \& JS Kotiaho. 2001. Fluctuating asymmetry. En: Group Nature Publishing (ed). Encyclopedia of Life Sciences, pp. 1-5. Macmillan Publishers, London.

van Valen L. 1962. A study of fluctuating asymmetry. Evolution 16(2): 125-142.

Via S, R Gomulkiewics, G de Jong, SM Scheiner, CD Schlichting \& PH van Tienderen. 1995. Adaptative phenotypic plasticity: consensus and controversy. Trends in Ecology and Evolution 10(5): 212-217.

Voets J, W Talloen, $\mathrm{T}$ de Tender, S van Dongen, A Covaci, R Blust \& L Bervoets. 2006. Microcontaminant accumulation, physiological condition and bilateral asymmetry in zebra mussels (Dreissena polymorpha) from clean and contaminated surface waters. Aquatic Toxicology 79(3): 213-225.

Recibido el 18 de octubre de 2007 y aceptado el 11 de enero de 2008 